\title{
Application of Mathematica Software to Solve Pulp Washing Model
}

\author{
Jitender Kumar, ${ }^{1}$ Ishfaq A. Ganaie, ${ }^{2}$ and Vijay K. Kukreja ${ }^{2}$ \\ ${ }^{1}$ Department of Applied Sciences, BGIET, Sangrur, 148001 Punjab, India \\ ${ }^{2}$ Department of Mathematics, SLIET, Longowal, 148106 Punjab, India \\ Correspondence should be addressed to Vijay K. Kukreja; vkkukreja@gmail.com
}

Received 29 August 2013; Accepted 9 October 2013

Academic Editors: J. J. Rodriguez and A. M. Seayad

Copyright (c) 2013 Jitender Kumar et al. This is an open access article distributed under the Creative Commons Attribution License, which permits unrestricted use, distribution, and reproduction in any medium, provided the original work is properly cited.

\begin{abstract}
The removal of the bulk liquor surrounding the pulp fibers using less concentrated liquor is known as pulp washing. In the present study, a pulp washing model involving diffusion-dispersion through packed beds of finite length is presented. Separation of variables is applied to solve system of governing partial differential equations and the resulting equations are solved using Mathematica. Results from the present case are compared with those of previous investigators. The present case is giving better results than the previous investigators.
\end{abstract}

\section{Introduction}

The objective of pulp and paper industry to produce its target production with high efficiency and less environmental load can only be met by initiating a meticulously planned research on mathematical methods. Pulp washing plays an important role in reduction of black liquor solids in the pulp being carried forward for further processing. The efficiency of washing depends on the degree of mixing, rate of desorption, diffusion-dispersion of dissolved solids, and chemicals from the fibrous matrix. Modeling of pulp washing is done mainly using three approaches, namely, (a) process modeling (b) physical modeling, and (c) statistical modeling.

A complete review of the various process models used to describe pulp washing has been presented by [1]. Initially researchers like in $[2,3]$ proposed the models based on axial dispersion. Pellett [4] introduced a mathematical model combining the effects of particle diffusion and axial dispersion. A detailed model related to mass transfer in fibrous particle was given by [5]; it was also restricted for axial dispersion only. Comprehensive models involving physical features of the fibers such as fiber porosity and fiber radius were presented by $[6,7]$.

Extensive study of axial dispersion model has been carried out by [8-26]. The model has been solved using analytic and numerical techniques like Laplace transform technique $[2-4,10,15,23,26]$, finite difference technique [25], orthogonal collocation method $[5,7,12]$, orthogonal collocation on finite elements [6, 20,21], Galerkin/Petrov Galerkin method $[8,19]$, Hermite collocation method by $[11,17,24]$ and Spline collocation method [13].

The accuracy of the analytic solution undoubtedly exceeds the limit of applicability of the theory to real situations. Moreover, it is highly desirable to have a simple and consistent model of the transport phenomenon based on essential features of real situation. Keeping this modest goal in mind axial dispersion model is solved along with linear adsorption isotherm. The method of separation of variables is first applied on partial differential equation and then Laplace transform is taken of the equations. Finally the mathematical expressions are solved using Mathematica software to obtain solute concentration at any location and time.

\section{Model Based on Axial Dispersion}

The displacement washing model based on the axial dispersion and particle diffusion describing the washing zone is given by

$$
\frac{\partial c}{\partial t}+u \frac{\partial c}{\partial z}+\mu \frac{\partial n}{\partial t}=D_{L} \frac{\partial^{2} c}{\partial z^{2}}
$$


with adsorption isotherm

$$
n=k c .
$$

This equation represents the basis for the mathematical models of displacement washing, where $t$ is the time from the commencement of the displacement, $z$ is the distance from the point of introduction of the displacing fluid, $c=c(z, t)$ is the solute concentration, $D_{L}$ is longitudinal dispersion coefficient, $u$ is the average interstitial velocity of the fluid, and $L$ is thickness of the packed bed.

On account of unusual nature of displacement process, appropriate boundary conditions have been extensively discussed in the literature $[2,6,27,28]$. Accordingly, the boundary conditions at the inlet and outlet of the bed are

$$
\begin{array}{cl}
c=c_{s} & \text { at } z=0, \\
\frac{\partial c}{\partial z}=0 & \text { at } z=L
\end{array}
$$

and initial condition is given by

$$
c(z, 0)=n(z, 0)=c_{i} \quad \text { for } 0<t<\frac{L}{u} .
$$

Conversion of Model into Dimensionless Form. Equations (1) to (4) can be put in dimensionless form using dimensionless variables:

$$
\begin{aligned}
& C=\frac{c-c_{s}}{c_{i}-c_{s}} ; \quad N=\frac{n-c_{s}}{c_{i}-c_{s}} ; \\
& Z=\frac{z}{L} ; \quad T=\frac{u t}{(1+\mu k) L} .
\end{aligned}
$$

The dimensionless time, $T$, corresponds physically to the number of pore displacements introduced into the medium since the start of the experiment.

By these means, (1) reduces to

$$
\frac{\partial C}{\partial T}+\frac{\partial C}{\partial Z}=\frac{1}{\operatorname{Pe}} \frac{\partial^{2} C}{\partial Z^{2}}
$$

where Pe $=u L / D_{L}$ is the Peclet number. The boundary conditions are now of the form

$$
\begin{array}{lll}
C(0, T)=0 \quad \text { at } Z=0 \quad \text { for } T>0, \\
\frac{\partial C(1, T)}{\partial Z}=0 \quad \text { at } Z=1 & \text { for } T>0
\end{array}
$$

while the initial condition is

$$
C=1 \quad \text { at } T=0 \quad \text { for } 0<Z \leq 1 .
$$

Now, our main aim is to estimate $C=C(Z, T)$ satisfying (7)(9), which will eventually lead to exit solute concentration $C_{e}=C_{e}(T)=C(1, T)$.

\section{Solution of Model}

Method of separation of variables is applied to solve (6). This method transforms the PDE into a system of ODEs, each of which depends only on one of the functions and the solution is given as product of the functions.

Equation (6) can be separated in terms of variables $Z$ and $T$ by assuming that $C(Z, T)=X(Z) Y(T)$ and then substituting $\partial C / \partial Z=X^{\prime} Y$ and $\partial C / \partial T=X Y^{\prime}$ in it, as follows:

$$
\frac{X^{\prime \prime}-\mathrm{Pe} X^{\prime}}{X}=\frac{\operatorname{Pe} Y^{\prime}}{Y}=-p^{2} \text { (constant) } .
$$

Individual solutions of expression (10) are given by

$$
\begin{gathered}
X(Z)=\exp \left(\frac{\mathrm{Pe} Z}{2}\right)\left[c_{1} \cos (\alpha Z)+c_{2} \sin (\alpha Z)\right], \\
Y(T)=c_{3} \exp \left(-\frac{p^{2} T}{\mathrm{Pe}}\right) .
\end{gathered}
$$

Application of boundary condition $X=0$ at $Z=0$ in (11) gives $c_{1}=0$ and the boundary condition $\partial X / \partial Z=0$ at $Z=1$ gives $\operatorname{Pe} \tan \alpha+2 \alpha=0$.

Therefore the solution $C(Z, T)=X(Z) Y(T)$ is given by

$$
C(Z, T)=A \exp \left(\frac{\mathrm{Pe} Z}{2}\right) \sin (\alpha Z) \exp \left(-\frac{p^{2} T}{\mathrm{Pe}}\right) .
$$

Equation Petan $\alpha+2 \alpha=0$ is a transcendental equation; it will have infinite many root; therefore the solution (13) will depend on $n$; that is,

$$
\begin{aligned}
C(Z, T) \sim C_{n}(Z, T)= & X_{n}(Z) Y_{n}(T) \\
= & A_{n} \exp \left(\frac{\operatorname{Pe} Z}{2}\right) \sin \left(\alpha_{n} Z\right) \\
& \times \exp \left(-\frac{p_{n}^{2} T}{\mathrm{Pe}}\right) .
\end{aligned}
$$

Using the principle of superposition, we get

$$
\begin{aligned}
C(Z, T) & =\sum_{n=1}^{\infty} C_{n}(Z, T) \\
& =\sum_{n=1}^{\infty} A_{n} \exp \left(\frac{\mathrm{Pe} Z}{2}\right) \sin \left(\alpha_{n} Z\right) \exp \left(-\frac{p_{n}{ }^{2} T}{\mathrm{Pe}}\right) .
\end{aligned}
$$

Applying the initial condition $C(Z, 0)=1$, we find that

$$
\sum_{n=1}^{\infty} A_{n} \sin \left(\alpha_{n} Z\right)=\exp \left(-\frac{\mathrm{Pe} Z}{2}\right) ;
$$

therefore $A_{n}$ represents the Strum-Liouville problem for $\exp (-\mathrm{Pe} Z / 2)$ and is given by

$$
A_{n}=\frac{\int_{0}^{1} e^{-\mathrm{Pe} Z / 2} \sin \left(\alpha_{n} Z\right) d Z}{\int_{0}^{1} \sin ^{2}\left(\alpha_{n} Z\right) d Z} .
$$


Finally, the solute concentration at any location and time in the bed can be written as

$$
\begin{aligned}
C(Z, T)= & \sum_{n=1}^{\infty} \frac{\int_{0}^{1} e^{-\mathrm{Pe} Z / 2} \sin \left(\alpha_{n} Z\right) d Z}{\int_{0}^{1} \sin ^{2}\left(\alpha_{n} Z\right) d Z} \\
& \times \exp \left(\frac{\mathrm{Pe} Z}{2}-\frac{p_{n}^{2} T}{\mathrm{Pe}}\right) \sin \left(\alpha_{n} Z\right),
\end{aligned}
$$

where $\alpha_{n}(n=1,2,3, \ldots)$ are the positive roots, taken in order of increasing magnitude, of the transcendental equation Pe $\tan \alpha_{n}+2 \alpha_{n}=0$ and $p_{n}=\sqrt{\left(4 \alpha_{n}^{2}+\mathrm{Pe}^{2}\right) / 4}$.

It is important to mention that (6) is also solved analytically by Grahs [5] and Kukreja [14]. Their solutions are given below.

Applying separation of variables, Grahs [5] found the concentration of solute as

$$
\begin{aligned}
C(Z, T)= & \exp \left\{\frac{\mathrm{Pe}}{2}\left(Z-\frac{T}{2}\right)\right\} \\
& \times\left[\sum_{n=1}^{\infty} \frac{4 \alpha_{n}^{2} \sin \left(\alpha_{n} Z\right) \exp \left(-\alpha_{n}^{2} T / \mathrm{Pe}\right)}{\left\{2 \alpha_{n}-\sin \left(2 \alpha_{n}\right)\right\}\left((\mathrm{Pe} / 4)+\alpha_{n}^{2}\right)}\right]
\end{aligned}
$$

where $\alpha_{n}$ are the positive roots of Pe $\tan \alpha_{n}+4 \alpha_{n}=0$.

Using Laplace transform, Kukreja [14] found the concentration of solute as

$$
\begin{aligned}
\frac{c-c_{s}}{c_{i}-c_{s}}= & \sum_{n=1}^{\infty} 4 \alpha_{n}^{2} \exp \left(p_{n} T+\frac{\mathrm{Pe} Z}{2}\right) \\
& \times \sum_{n=1}^{\infty} \frac{\left[\mathrm{Pe} \sin \left\{(Z-1) \alpha_{n}\right\}-2 \alpha_{n} \cos \left\{(Z-1) \alpha_{n}\right\}\right]}{\operatorname{Pe} p_{n}\left(\mathrm{Pe}^{2}+4 \alpha_{n}^{2}+2 \mathrm{Pe}\right) \sin \left(\alpha_{n}\right)}
\end{aligned}
$$

where Pe $\tan \alpha_{n}+2 \alpha_{n}=0$ and $p_{n}=-\left(4 \alpha_{n}^{2}+\mathrm{Pe}^{2}\right) / 4 \mathrm{Pe}$.

The solutions given by (18)-(20) are complex and time consuming. These equations are evaluated using Mathematica software. This technique is novel and elegant and can be conveniently handled by any nonmathematician also.

\section{Results and Discussion}

Solution of (18)-(20) are obtained using Mathematica by taking 22 mesh points within the dimensionless time and also in dimensionless distance $x$. For $\mathrm{Pe}=1$, the surface plots of the dimensionless concentration with respect to dimensionless distance $(0 \leq x \leq 1)$ and dimensionless time $(0 \leq T \leq 1.2)$ are shown in Figures 1, 2, and 3, respectively, for the present case (18), Grahs (19), and Kukreja (20). It can be observed from Figure 1 that the surface plot of (18) is very smooth at $T=0$, whereas for (19) and (20) fluctuation is observed.

In Figures 4 to 6, exit solute concentration obtained from (18)-(20) is plotted, respectively. In Figure 5, the solution profile of (19) is starting from 1.3 approximately, which is highly impossible in pulp washing system, as the dimensionless concentration can never exceed 1 . Smoothest profile can be seen in case of Figure 4.

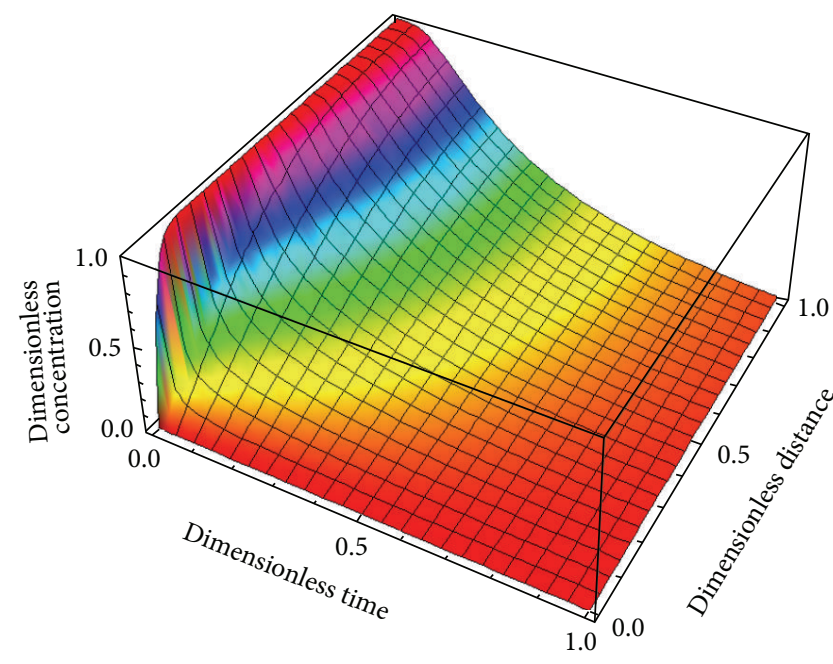

FIGURE 1: Surface plot for the present case represented by (18).

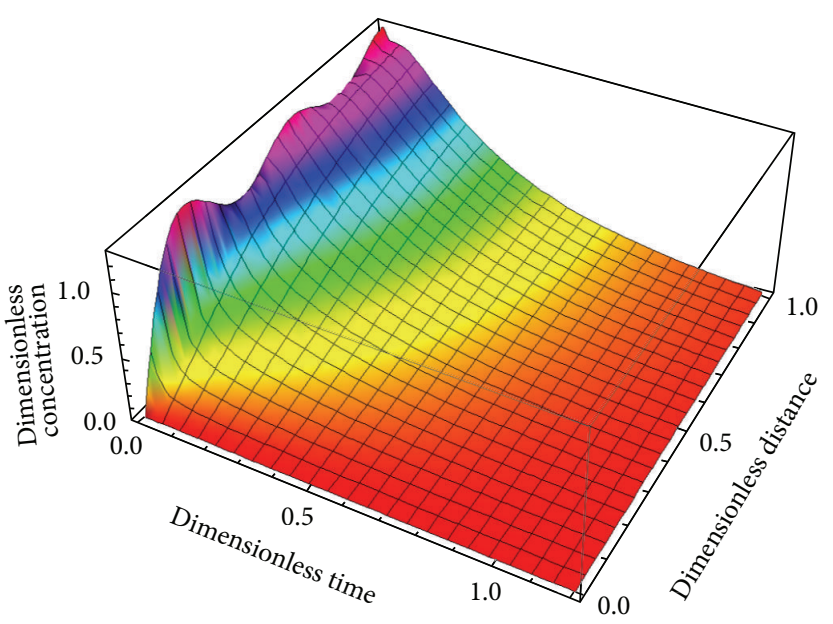

Figure 2: Surface plot for Grahs [5] represented by (19).

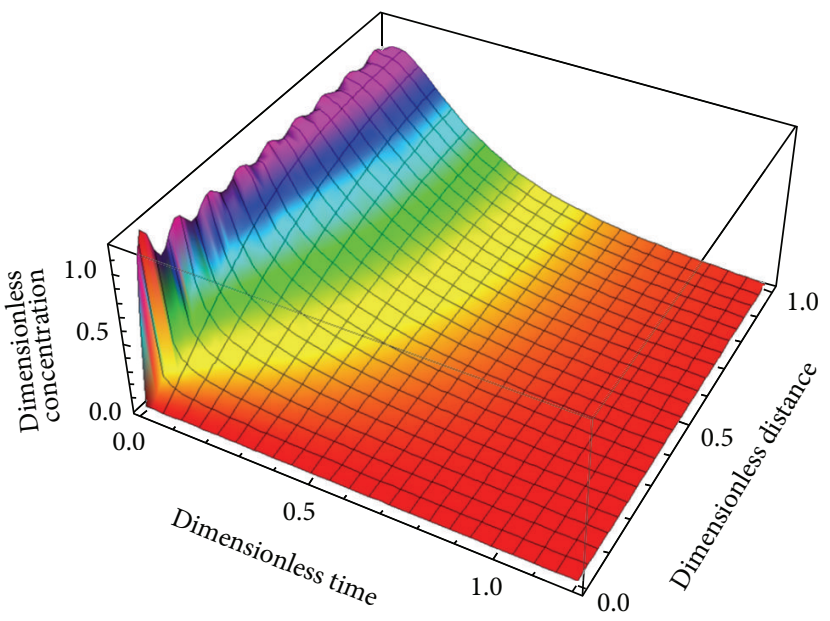

FIgURE 3: Surface plot for Kukreja [15] represented by (20). 


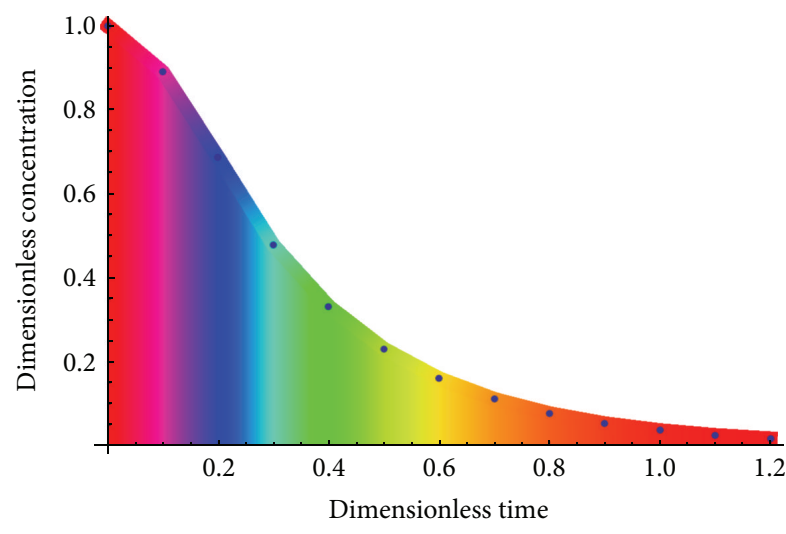

FIGURE 4: Exit solute concentration profile for present case.

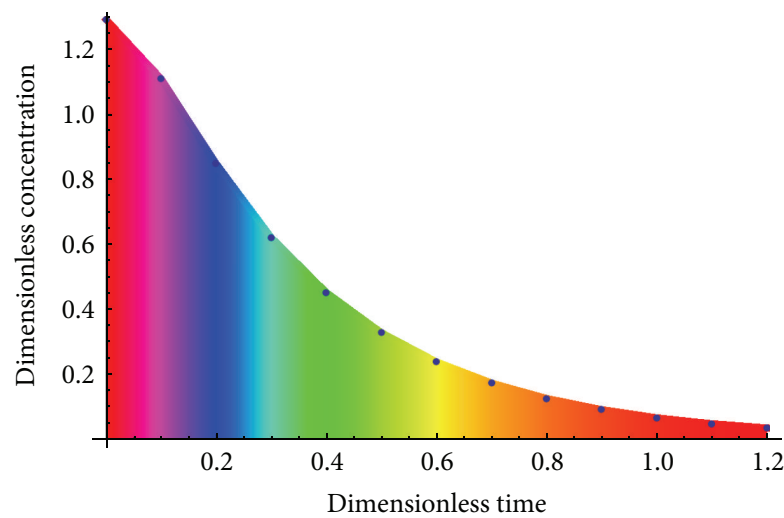

FIGURE 5: Exit solute concentration profile by Grahs [5].

In Figures 7 and 8, the absolute error obtained from (19) and (20) from (18) is plotted. The magnitude of error in case of (19) is 4 to 5 times higher the (20). This indicates more deviation between the present case and Grahs [5] as compared to Kukreja [14].

The results from the three investigators are summarized in Table 1. At $T=0$ the error with Kukreja [14] is $2.92 \%$, whereas with Grahs [5] it is $29.13 \%$; ideally, these should have been $0.0 \%$. Similarly, at $T=0.1$, the error is found to be $6.25 \%$ while comparing the results with Kukreja [14] and for Grahs [5] it is $12.23 \%$, which is again very high. It can further be seen in Table 1 that at $T=0.5$ onwards the error is $0.0 \%$ with Kukreja [14] whereas with Grahs [5] it is reducing but persists continuously. Hence, this comparison shows that results of Kukreja [14] are matching with present case but those of Grahs [5] are showing significant error.

\section{Conclusion}

The investigation, based on diffusion model of longitudinal mixing in beds of finite length, is applicable to displacement washing with axial dispersion and particle diffusion. The present solution of the model as well as the solution proposed by Grahs [5] and Kukreja [14] involves complicated expressions. The application of Mathematica makes it convenient

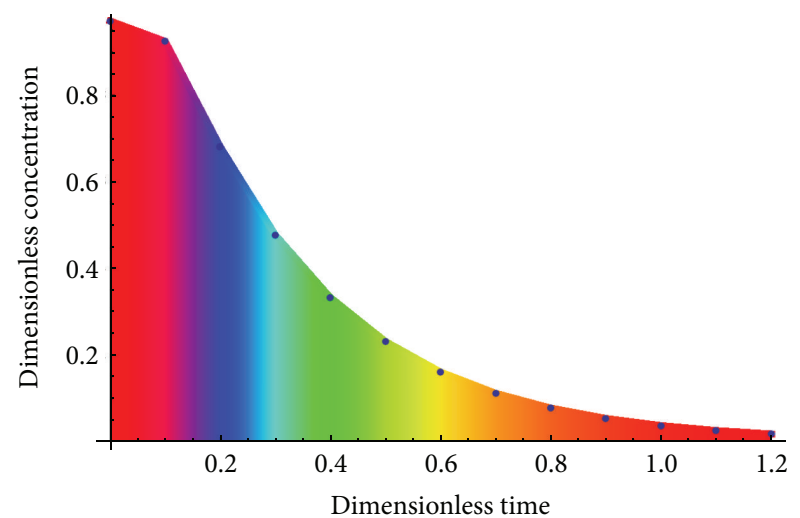

Figure 6: Exit solute concentration profile by Kukreja [14].

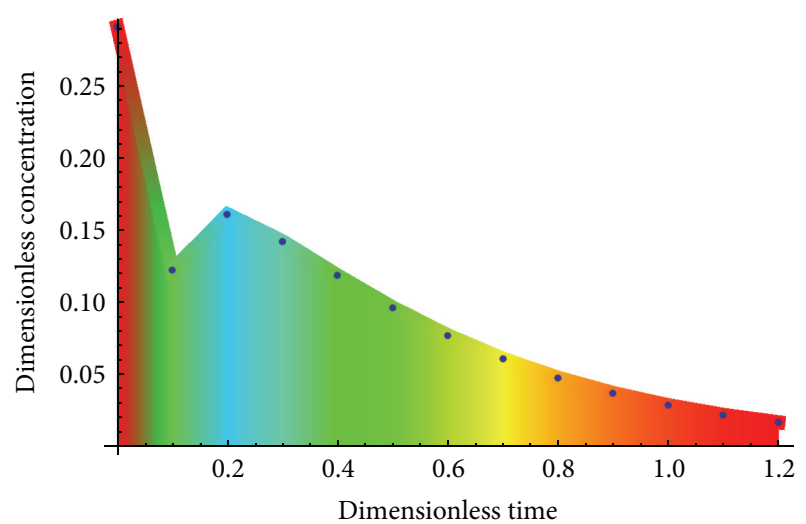

Figure 7: Absolute error between present case and Grahs [5].

TABLE 1: Comparison of solution of Grahs [5] and Kukreja [14] with the present case.

\begin{tabular}{cccccc}
\hline Time & $\begin{array}{c}\text { Present } \\
\text { case }\end{array}$ & $\begin{array}{c}\text { Grahs } \\
{[5]}\end{array}$ & $\begin{array}{c}\text { Kukreja } \\
{[14]}\end{array}$ & $\begin{array}{c}\text { \% error } \\
\text { with [5] }\end{array}$ & $\begin{array}{c}\text { \% error } \\
\text { with [14] }\end{array}$ \\
\hline 0.00 & 1.0000 & 1.2913 & 0.9708 & 29.13 & 2.92 \\
0.10 & 0.9864 & 0.1087 & 0.9239 & 12.23 & 6.25 \\
0.20 & 0.6866 & 0.8477 & 0.6805 & 16.11 & 0.61 \\
0.30 & 0.4779 & 0.6203 & 0.4773 & 14.24 & 0.06 \\
0.40 & 0.3326 & 0.4512 & 0.3326 & 11.86 & 0.00 \\
0.50 & 0.2315 & 0.3279 & 0.2315 & 09.64 & 0.00 \\
0.60 & 0.1611 & 0.2382 & 0.1611 & 07.71 & 0.00 \\
0.70 & 0.1122 & 0.1731 & 0.1122 & 06.09 & 0.00 \\
0.80 & 0.0781 & 0.1258 & 0.0781 & 04.77 & 0.00 \\
0.90 & 0.0543 & 0.0914 & 0.0543 & 03.71 & 0.00 \\
1.00 & 0.0378 & 0.0664 & 0.0378 & 02.86 & 0.00 \\
1.10 & 0.0263 & 0.0482 & 0.0263 & 02.19 & 0.00 \\
1.20 & 0.0183 & 0.0350 & 0.0183 & 01.67 & 0.00 \\
\hline
\end{tabular}

to evaluate these expressions. The present results are more accurate than the output provided by Grahs [5] and Kukreja [14]. The algorithms based on Mathematica are novel and easy to set up. Therefore, the present technique provides a good 


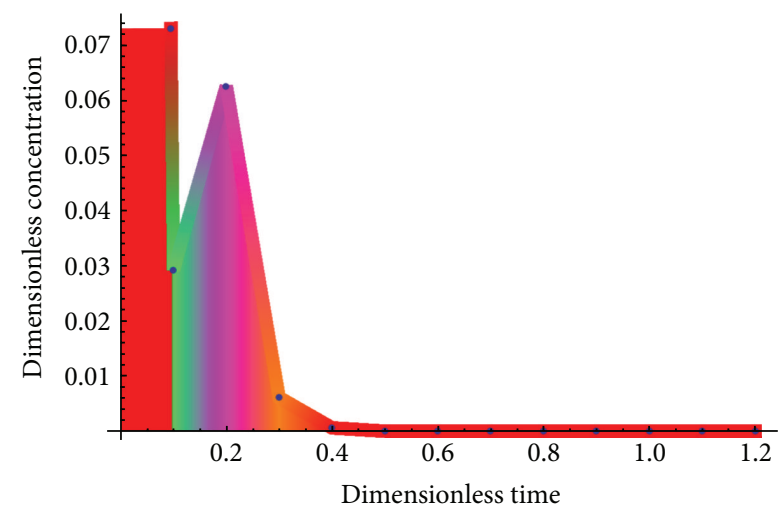

FIGURE 8: Absolute error between the present case and Kukreja [14].

alternate to the available techniques for dealing with such type of problems.

\section{Nomenclature}

$c:$ Concentration of the solute in the liquor, $\mathrm{kg} / \mathrm{m}^{3}$

$c_{i}$ : Initial solute concentration, $\mathrm{kg} / \mathrm{m}^{3}$

$c_{s}$ : Solute concentration of incoming fluid, $\mathrm{kg} / \mathrm{m}^{3}$

$c_{e}$ : Exit solute concentration from the bed, $\mathrm{kg} / \mathrm{m}^{3}$

$D_{L}$ : Longitudinal dispersion coefficient, $\mathrm{m}^{2} / \mathrm{s}$

$L: \quad$ Cake thickness, $m$

$n$ : Concentration of the solute in the fiber, $\mathrm{kg} / \mathrm{m}^{3}$

$t:$ Time, $\mathrm{s}$

$u$ : Liquor speed in cake pores, $\mathrm{m} / \mathrm{s}$

$z$ : Distance from point of introduction of solvent, $\mathrm{m}$

$\varepsilon: \quad$ Porosity of packed bed

$\mu$ : Ratio of porosity, $(1-\varepsilon) / \varepsilon$.

\section{Acknowledgments}

This work is supported by NBHM, Mumbai, India, in the form of research projects $2 / 48(14) / 2009 / \mathrm{R} \& \mathrm{D}-\mathrm{II} / 2806$. The JRF provided to Mr. Ishfaq A. Ganaie by NBHM, Mumbai, is thankfully acknowledged.

\section{References}

[1] M. Pekkanen and H. V. Norden, "Review of pulp washing models," Paperi Ja Puu, vol. 67, pp. 689-696, 1985.

[2] H. Brenner, "The diffusion model of longitudinal mixing in beds of finite length. Numerical values," Chemical Engineering Science, vol. 17, no. 4, pp. 229-243, 1962.

[3] W. R. Sherman, "The movement of a soluble material during the washing of a bed of packed solids," AIChE Journal, vol. 10, pp. 855-860, 1964.

[4] G. L. Pellett, "Longitudinal dispersion, intra particle diffusion and liquid-phase mass transfer during flow through multi particle systems," TAPPI Journal, vol. 49, pp. 75-82, 1966.

[5] L. E. Grahs, Washing of cellulose fibres: analysis of displacement washing operation [Ph.D. dissertation], Department of Chemical Engineering, Chalmers University of Technology, Gothenburg, Sweden, 1974.
[6] S. Arora, S. S. Dhaliwal, and V. K. Kukreja, "Simulation of washing of packed bed of porous particles by orthogonal collocation on finite elements," Computers \& Chemical Engineering, vol. 30, no. 6-7, pp. 1054-1060, 2006.

[7] N. S. Raghavan and D. M. Ruthven, "Numerical simulation of a fixed-bed adsorption column by the method of orthogonal collocation," AIChE Journal, vol. 29, no. 6, pp. 922-925, 1983.

[8] M. Al-Jabari, A. R. P. van Heiningen, and T. G. M. van de Ven, "Modeling the flow and the deposition of fillers in packed beds of pulp fibres," Journal of Pulp and Paper Science, vol. 20, no. 9, pp. J249-J253, 1994.

[9] S. Arora and F. Potůček, "Modelling of displacement washing of packed bed of fibers," Brazilian Journal of Chemical Engineering, vol. 26, no. 2, pp. 385-393, 2009.

[10] B. V. Babu and A. S. Chaurasia, "Pyrolysis of biomass: Improved models for simultaneous kinetics and transport of heat, mass and momentum," Energy Conversion and Management, vol. 45, no. 9-10, pp. 1297-1327, 2004.

[11] K. D. Edoh, R. D. Russell, and W. Sun, "Computation of invariant tori by orthogonal collocation," Applied Numerical Mathematics, vol. 32, no. 3, pp. 273-289, 2000.

[12] L. T. Fan, G. K. C. Chen, and L. E. Erickson, "Efficiency and utility of collocation methods in solving the performance equations of flow chemical reactors with axial dispersion," Chemical Engineering Science, vol. 26, no. 3, pp. 378-387, 1971.

[13] B. Gupta and V. K. Kukreja, "Modelling \& simulation of packed bed of porous particles by orthogonal spline collocation method," Applied Mathematics \& Computation, vol. 219, pp. 2087-2099, 2012.

[14] V. K. Kukreja, Modeling of washing of brown stock on rotary vacuum washer [Ph.D. dissertation], Department of Pulp and Paper Technology, University of Roorkee, Roorkee, India, 1996.

[15] V. K. Kukreja and A. K. Ray, "Mathematical modeling of a rotary vacuum washer used for pulp washing: a case study of a lab scale washer," Cellulose Chemistry \& Technology, vol. 43, no. 1-3, pp. 25-36, 2009.

[16] A. Kumar, D. K. Jaiswal, and N. Kumar, "Analytical solutions of one-dimensional advection-diffusion equation with variable coefficients in a finite domain," Journal of Earth System Science, vol. 118, no. 5, pp. 539-549, 2009.

[17] C. P. Leão and A. E. Rodrigues, "Transient and steady-state models for simulated moving bed processes: numerical solutions," Computers \& Chemical Engineering, vol. 28, no. 9, pp. 1725-1741, 2004.

[18] H.-T. Liao and C.-Y. Shiau, "Analytical solution to an axial dispersion model for the fixed-bed adsorber," AIChE Journal, vol. 46, no. 6, pp. 1168-1176, 2000.

[19] F. Liu and S. K. Bhatia, "Application fo Petrov-Galerkin methods to transient boundary value problems in chemical engineering: adsorption with steep gradients in bidisperse solids," Chemical Engineering Science, vol. 56, no. 12, pp. 3727-3735, 2001.

[20] Y. Liu and E. W. Jacobsen, "On the use of reduced order models in bifurcation analysis of distributed parameter systems," Computers \& Chemical Engineering, vol. 28, no. 1-2, pp. 161-169, 2004.

[21] Z. Ma and G. Guiochon, "Application of orthogonal collocation on finite elements in the simulation of non-linear chromatography," Computers \& Chemical Engineering, vol. 15, no. 6, pp. 415-426, 1991.

[22] F. Potůček, "Washing of pulp fibre bed," Collection of Czechoslovak Chemical Communications, vol. 62, no. 4, pp. 626-644, 1997. 
[23] A. Rasmuson and I. Neretnieks, "Exact solution of a model for diffusion in particles and longitudinal dispersion in packed beds," AIChE Journal, vol. 26, pp. 686-690, 1980.

[24] A. La Rocca and H. Power, "A double boundary collocation Hermitian approach for the solution of steady state convectiondiffusion problems," Computers and Mathematics with Applications, vol. 55, no. 9, pp. 1950-1960, 2008.

[25] L. M. Sun and F. Meunier, "An improved finite difference method for fixed-bed multicomponent sorption," AIChE Journal, vol. 37, no. 2, pp. 244-254, 1991.

[26] Y. Zheng and T. Gu, "Analytical solution to a model for the startup period of fixed-bed reactors," Chemical Engineering Science, vol. 51, no. 15, pp. 3773-3779, 1996.

[27] F. Shiraishi, "Highly accurate solution of the axial dispersion model expressed in S-system canonical form by Taylor series method," Chemical Engineering Journal, vol. 83, no. 3, pp. 175$183,2001$.

[28] M. K. Szukiewicz, "New approximate model for diffusion and reaction in a porous catalyst," AIChE Journal, vol. 46, no. 3, pp. 661-665, 2000. 

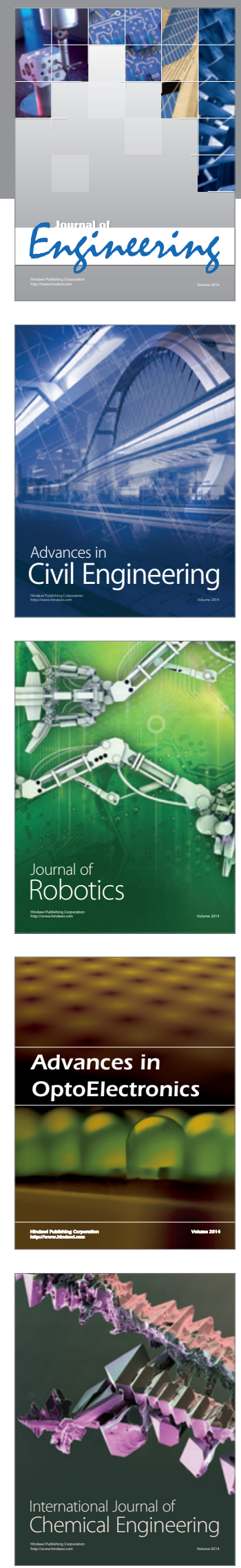

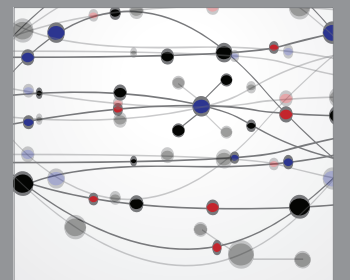

The Scientific World Journal
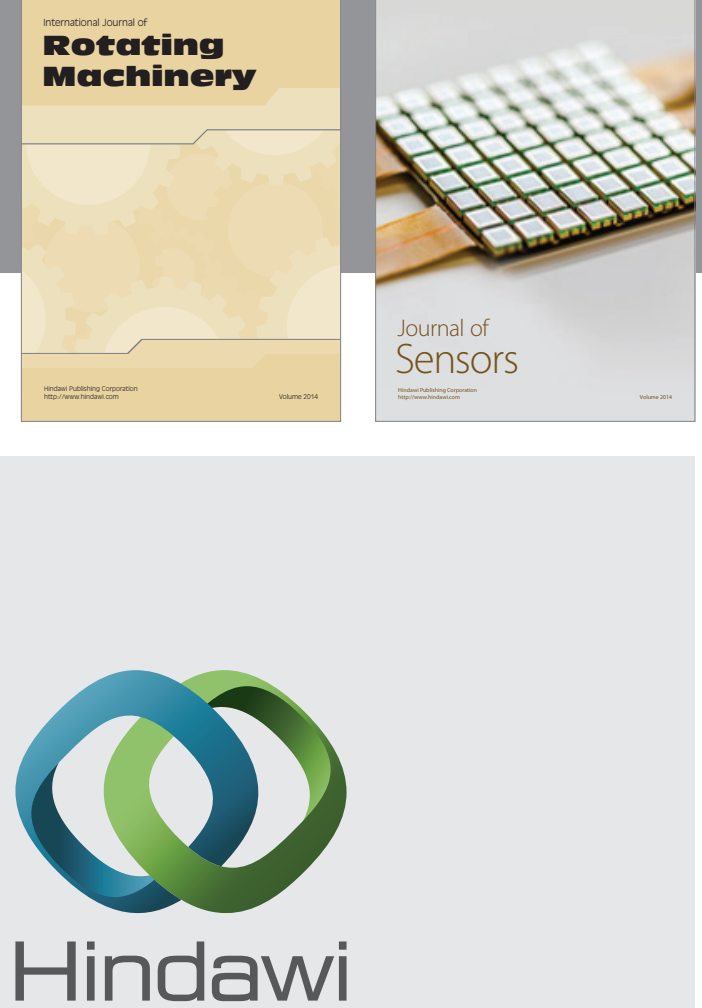

Submit your manuscripts at http://www.hindawi.com
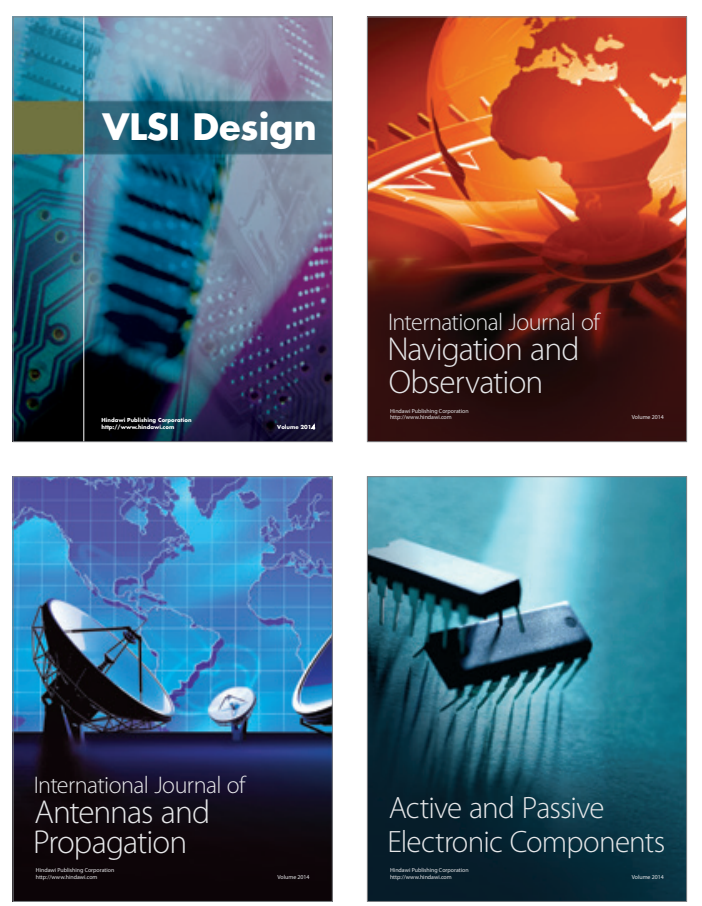
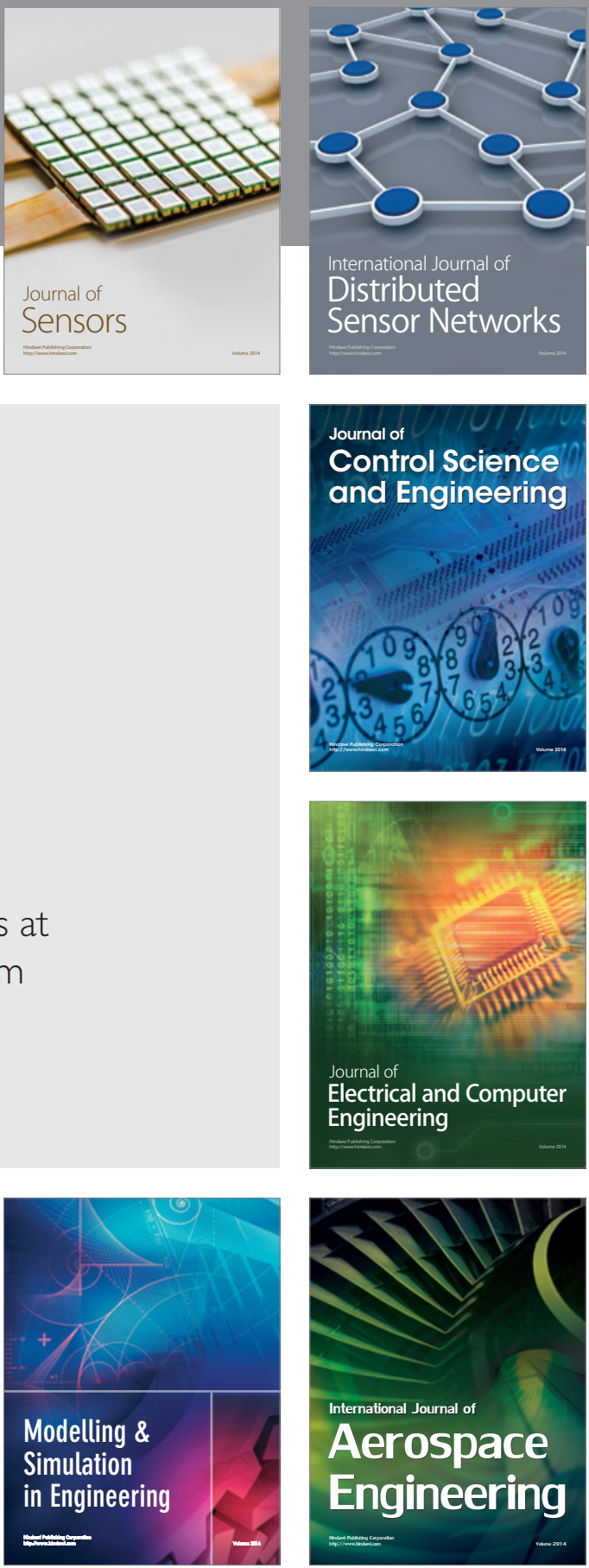

Journal of

Control Science

and Engineering
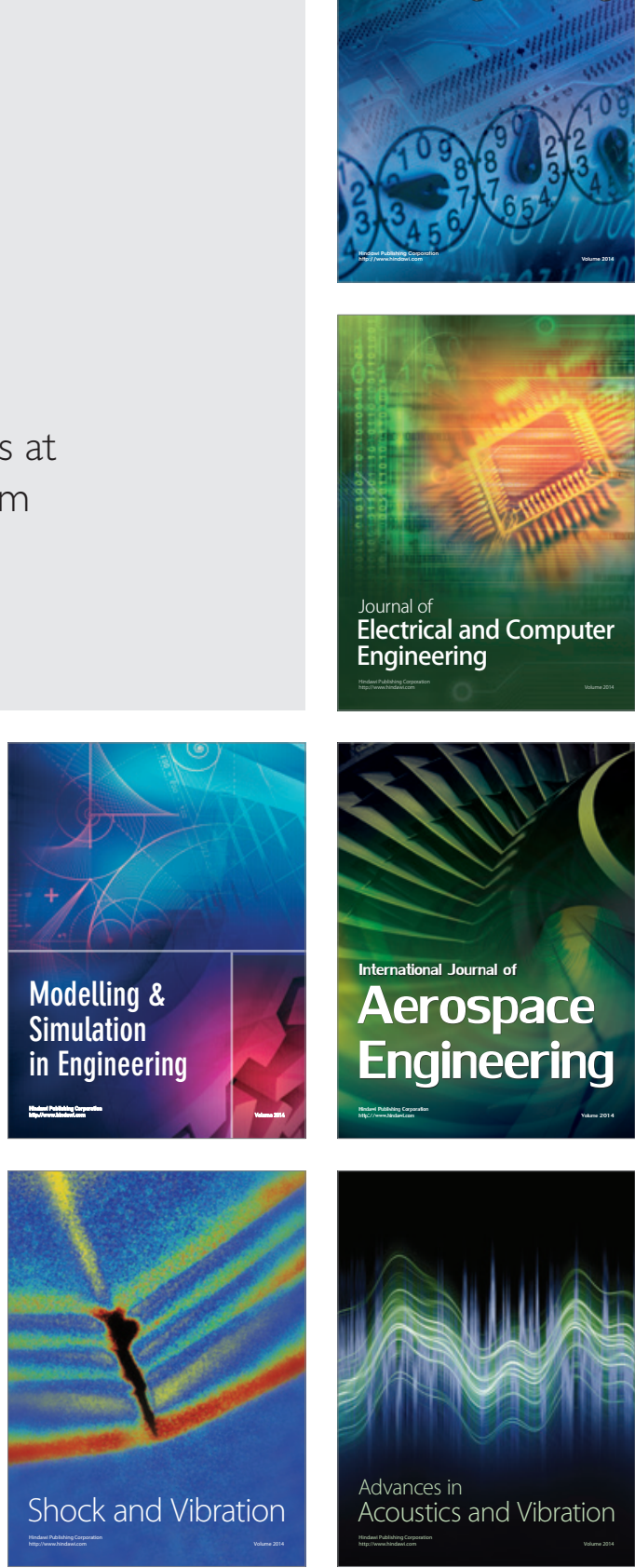